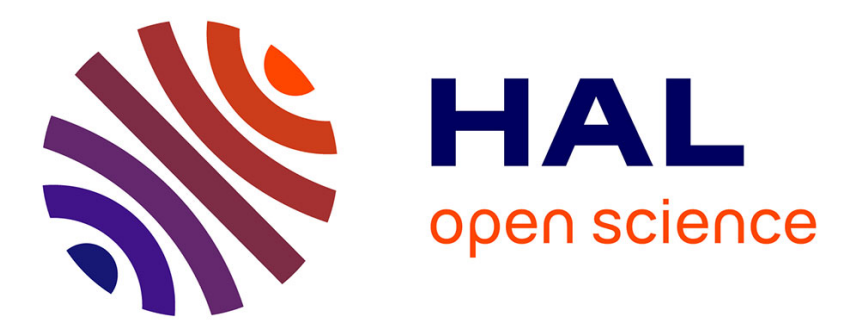

\title{
Politique industrielle en Émilie-Romagne: un modèle en quête de son avenir
}

\author{
Nicola Bellini, Mohammad-Saïd Darviche, Emmanuel Négrier
}

\section{To cite this version:}

Nicola Bellini, Mohammad-Saïd Darviche, Emmanuel Négrier. Politique industrielle en ÉmilieRomagne: un modèle en quête de son avenir. Pôle Sud - Revue de science politique de l'Europe méridionale, 1996, L'Europe du Sud est-elle en retard?, 5, pp.117-131. 10.3406/pole.1996.951 . hal02528276

\section{HAL Id: hal-02528276 \\ https://hal.science/hal-02528276}

Submitted on 24 Apr 2020

HAL is a multi-disciplinary open access archive for the deposit and dissemination of scientific research documents, whether they are published or not. The documents may come from teaching and research institutions in France or abroad, or from public or private research centers.
L'archive ouverte pluridisciplinaire HAL, est destinée au dépôt et à la diffusion de documents scientifiques de niveau recherche, publiés ou non, émanant des établissements d'enseignement et de recherche français ou étrangers, des laboratoires publics ou privés.

\section{(ㅇ)(1) $\$$}

Distributed under a Creative Commons Attribution - NonCommercial - NoDerivatives $\mid 4.0$ 


\section{Politique industrielle en Émilie-Romagne : un modèle en quête} de son avenir

Mr Nicola Bellini, Mohammad-Saïd Darviche, Mr Emmanuel Négrier

\section{Citer ce document / Cite this document :}

Bellini Nicola, Darviche Mohammad-Saïd, Négrier Emmanuel. Politique industrielle en Émilie-Romagne : un modèle en quête de son avenir. In: Pôle Sud, n5, 1996. L'Europe du Sud est-elle en retard ? pp. 117-131;

doi : https://doi.org/10.3406/pole.1996.951

https://www.persee.fr/doc/pole_1262-1676_1996_num_5_1_951

Fichier pdf généré le 29/03/2019 


\title{
Politique industrielle en Émilie-Romagne :
} un modèle en quête de son avenir

\author{
par Nicola Bellini \\ Scuola Superiore S. Anna \\ (Traduit de l'anglais par M.-S. Darviche et E. Négrier) \\ Pôle Sud $N^{\circ} 5$ - Novembre $1996-p p .117$ à 131
}

\begin{abstract}
Pendant longtemps la région Émilie-Romagne a été considérée, en Italie et au-delà, comme un modèle de développement économique, capable d'associer performance industrielle et paix sociale. La mise en place, dans les années 70 , par les autorités régionales, d'une agence de développement économique était le signe d'une volonté concertée d'optimisation du tissu industriel par la formation professionnelle d'une part, et la mise en réseau des petites entreprises pour améliorer l'innovation de l'autre. Avec les années 90 , le modèle commence à montrer des signes de faiblesse face à la montée du chômage, la nécessité de modernisation des infrastructures et aux problèmes liés à l'environnement. Néanmoins devant l'inexistence d'instruments fiables pour évaluer les effets du modele, il est difficile d'accepter les sévères critiques qui lui ont été adressées. En effet, sans être transposable tel quel, le modèle émilien demeure une expérience qui, tout en comportant des risques, reste à la fois originale et prometteuse quant aux capacités d'une politique industrielle décentralisée dont le pivot serait la région.
\end{abstract}

\section{Une introduction à l'économie politique d'Émilie-Romagne}

Au moins depuis l'essai de S. Brusco publié dans un numéro de $1982 \mathrm{du}$ Cambridge Journal of Economics (Brusco, 1982), "le modèle émilien" a été un label internationalement reconnu pour désigner la recette particulière (et peut-être unique) du succès économique de cette belle, industrieuse, lettrée et riche région italienne, parmi celles ayant un des niveaux les plus élevés d'Europe. Au cœur du "modèle émilien" il y a cette intégration entre petites entreprises que l'on retrouve (quoique partiellement) dans le reste de ce que l'on appelle la Troisième Italie.

Nous pouvons spécifier rapidement ce qui suit comme les caractéristiques dis- tinctives de l'économie d'ÉmilieRomagne :

- un appareil de production très fragmenté, mais néanmoins souvent très intégré dans des zones territorialement délimitées; il s'agit dans la plupart des cas de "purs" exemples de districts industriels marshalliens (Pyke, Becattini, Segenberger, 1990);

- une tradition profondément ancrée d'intégration équilibrée entre des activités agriculturelles et manufacturières et de diverses industries ;

- une structure industrielle orientée vers l'exportation ;

- une division du travail, au sein d'un cycle de production unique, entre des compagnies indépendantes connectées les unes aux autres par des relations de production de quasi-marché ; 


\section{Pôle Sud $N^{\circ} 5$}

- l'absence d'une grande métropole et, au contraire, la présence d'un grand nombre de petites zones urbaines territorialement dispersées' ;

- enfin, dernière caractéristique et non des moindres, une remarquable stabilité des relations industrielles, réduisant l'impact des conflits et tensions liés au processus de modernisation, et allant de pair avec un soutien relativement efficace des autorités politiques aussi bien pour les activités économiques que pour la protection sociale ${ }^{2}$.

En fait, l'évidence du succès économique ne donne qu'une explication partielle de la force et de l'influence du "modèle émilien". Ce dernier était (et demeure) une combinaison extrêmement fascinante entre gouvernement progressiste, intégration sociale et succès entrepreneurial. (Il ne s'agit pas seulement d'un succès : pour beaucoup, c'est un modèle de développement économique plus authentiquement capitaliste que ce que l'on trouve dans des zones dominées par de grandes corporations oligopolistiques). Pour le Parti Communiste Italien, qui domine l'arène politique locale depuis la guerre, la diversité d'Émilie-Romagne pouvait préfigurer ce qu'aurait pu être un gouvernement de gauche dans le pays : un gouvernement fondé sur une large coalition (comme en Émilie-Romagne la coalition entre ville et campagne, entre prolétariat urbain et travailleurs agricoles et mezzadri), d'une grande efficacité et d'une stabilité à toute épreuve. Ces deux caractéristiques étaient censées contribuer largement au succès économique.

Le district industriel, véritable pierre angulaire du "modèle", était passé d'un concept analytique et d'une réalité historique (un modele différent de développement dans le respect du noyau dur de l'industrialisation italienne) à une sorte d'idéologie socio-économique : l'alternative au capitalisme conservateur et centralisé dominé par les grandes compagnies. Cet aspect idéologique, souvent négligé ${ }^{3}$, est confirmé par le fait qu'une telle auto-représentation est inapplicable à la totalité d'une Émilie-Romagne qui est loin d'être une réalité homogène. En fait, le "modèle émilien" s'applique presque exclusivement à la zone centrale (c'est-à-dire les provinces de Bologne, Modène et Reggio Emilia), où la structure districtale est plus clairement établie et où, parallèlement, l'hégémonie du $\mathrm{PCI}$ a été plus profondément enracinée. Au contraire, les autres zones (la province de Ferrare dans le Nord-Est ; la Romagne à l'Est ; les provinces de Piacenza et de Parme à l'Ouest) présentent une variété de niveaux de développement, de structures économiques, de connotations politiques, etc. En outre, du fait de la traditionnelle faiblesse de Bologne comme véritable centre métropolitain, la gravitation géographique, économique et culturelle de ces zones "périphériques" contrarie le plus souvent l'intégration des intérêts économiques et l'homogénisation du paysage socio-politique.

Les quinze dernières années de l'Émilie ont été marquées par une bonne performance économique mais, en quelque sorte, moins exceptionnelle. La restructuration et l'innovation dans les années 80 ont apparemment surtout bénéficié aux grandes compagnies. La restructuration des grands 


\section{Politique industrielle en Émilie-Romagne}

groupes et l'évolution du jeu de la compétition, qui ont de plus en plus récompensé la globalisation des marchés et l'internationalisation des entreprises, ont mis en évidence l'inéluctable faiblesse des plus petites. En plus des nouvelles réalisations en matière de spécialisation et de flexibilité, les avantages liés à la taille ont particulièrement concerné les fonctions financières, la recherche, le marketing et la présence directe sur les marchés étrangers. Ainsi, les positions compétitives acquises par les plus petites firmes sont devenues instables.

En fait, la remarquable performance de l'économie d'Émilie-Romagne dans les difficiles années 70 ne s'est pas reproduite durant la décennie suivante. L'Italie dans son ensemble et d'autres régions importantes, comme la Vénétie et la Lombardie, ont dépassé en performance l'ÉmilieRomagne.

Incontestablement, le système de production de la région est fortement impliqué dans cette transformation; des tendances positives peuvent même permettre une appréciation renouvelée de la formule du district (Franchi, 1994), en dépit d'une critique grandissante (bien qu'elle ait donné lieu à débat) concernant les trop faibles performances supposées des compagnies italiennes en matière d'innovation. En fait, les entreprises du district industriel peuvent demeurer petites et même particulièrement performantes, mais appartenir en réalité à de nouvelles structures de type hiérarchique.

Néanmoins, le système de production régional continue à montrer des problèmes et difficultés non résolus qui peuvent être rapportés à quelques questions fondamentales :

- comment sortir des modèles traditionnels de capitalisme familial,

- comment gérer l'internationalisation de la production, et

- comment maintenir des taux d'innovation élevés?

Ainsi le débat tourne, une fois de plus, autour du rôle des petites et moyennes entreprises et leur besoin de croître. L'objectif (et dilemme) politique en ÉmilieRomagne devient alors de savoir comment renforcer les opportunités et augmenter les ressources pour la croissance sans bouleverser l'unique et délicat équilibre social et politique du modèle émilien ${ }^{4}$.

\section{Une chronologie}

Dans la chronologie de la politique industrielle en Émilie-Romagne, les années 70 représentent la période où la région, en tant qu'institution nouvellement établie, cherchait un rôle dans la promotion de l'économie, en accord avec le traditionnel rôle de soutien des administrateurs locaux "rouges". Pendant ces années, la planification régionale faisait preuve d'une foi optimiste dans la solidité économique d'Émilie-Romagne, déclarant les caractéristiques structurelles de l'économie régionale comme la raison de son remarquable succès dans la limitation de l'impact négatif de la crise nationale, spécialement en ce qui concerne le chômage.

C'est seulement à la fin des années 70 que les régions italiennes ont finalement reçu les pouvoirs leur permettant d'exprimer quelques-unes de leurs potentialités 


\section{Pôle Sud $N^{\circ} 5$}

gouvernementales. Les années 80 peuvent être décrites comme l'“âge d'or" de la politique industrielle régionale. La plupart des expériences qui seront discutées plus tard dans cet article datent de cette période.

Au moyen d'un certain nombre d'initiatives couronnées de succès, la politique industrielle a acquis une signification politique majeure dans la stratégie du gouvernement régional et de son parti dominant, le PCI. Visiblement la politique industrielle a permis de donner une identité précise au rôle de la nouvelle institution et d'élargir substantiellement le système des alliances sociales. Ainsi, par exemple, les centres de service aux entreprises, notamment grâce à leur succès technique, se sont progressivement érigés en institutions quasi-corporatistes au niveau local, en partie du fait qu'ils ont enregistré une participation grandissante de toutes les composantes du système de représentation patronale sous l'égide de la région. En même temps, l'expérience d'Émilie-Romagne, de manière plus distincte que dans les autres régions, séduisit intellectuels et hommes politiques en Italie et, au-delà, renforça son image de "diversité positive".

De nouveaux thèmes ont caractérisé le débat sur la politique industrielle au début des années 90 . Un pessimisme grandissant sur la poursuite du succès du "modèle" porta l'attention à la fois sur les difficultés des petites et moyennes entreprises face aux défis de l'innovation et de l'internationalisation, et sur le déficit infrastructurel d'Émilie-Romagnes.

Entre temps, dans l'arène politique, la faiblesse relative du PCI (futur PDS) conduisit, après les élections administratives de 1990, à la formation de gouvernements de coalition dans plusieurs villes et provinces et dans la région elle-même, le prix de l'alliance étant l'attribution de quelques-unes des positions les plus en vue (incluant la présidence de la région) aux membres du Parti Socialiste. L'impact potentiellement innovateur de ce fait s'avéra relativement modeste, à cause de la performance variable de ces gouvernements de coalition et à cause du déclin catastrophique du Parti Socialiste luimême, dans les années qui ont suivi, à la fois nationalement et localement. Néanmoins, d'importants secteurs de politiques, incluant la politique industrielle, ont été fortement marqués par des politiciens qui, à juste titre ou non, soit à cause de convictions profondes soit d'opportunités contingentes, se sont sentis déliés des traditions politiques héritées. Parallèlement, les associations patronales se sont senties libres des pratiques antérieures et capables de jouer les partis et les hommes politiques les uns contre les autres, profitant ainsi politiquement de l'instabilité politique.

Ces changements d'équilibre expliquent pour une large part l'événement le plus significatif de ces dernières années, à savoir la crise d'ERVET. Il s'agissait d'un processus long et pénible, provoqué par une vague impressionnante de critiques (seulement partiellement justifiées) initiée par la Confindustria locale, et qui conduisit par la suite à un renouveau profond de l'agence, suivant des lignes que l'on discutera plus loin. 


\section{Politique industrielle en Émilie-Romagne}

\section{Les institutions de la politique industrielle régionale}

Les normes contenues dans le DPR (Decreto del Presidente della Repubblica) $n^{\circ} 616$ de juillet 1977 activa l'institutionnalisation des régions et la décentralisation des compétences étatiques en vertu de l'article 117 de la Constitution. À l'exception des régions frontalières (qui font face à des problèmes ethniques spécifiques) et les îles, le "statut ordinaire" des régions n'accorde pas de mandat pour conduire les affaires industrielles. Cependant, ils ont les pouvoirs pour s'occuper du développement territorial, et la formation artisanale et professionnelle.

Récemment est intervenue une extension de facto de tels pouvoirs, basés sur des thèmes horizontaux, dont l'impact ne peut être limité à des secteurs spécifiques du dispositif de production, comme l'innovation, la qualité, la promotion de l'exportation, l'internationalisation et l'innovation financière. De plus, une dévolution limitée, mais loin d'être sans conséquences, est implicite dans la loi ( $n^{\circ} 317$ of 1991), soutenant l'innovation dans les petites et moyennes compagnies.

Ceci est un élément fondamental à saisir pour une analyse comparative. Les régions italiennes n'ont jamais commencé avec une batterie complète d'options politiques, mais ont évolué à travers un processus graduel d'acquisition d'outils politiques et d'objectifs, en saisissant les opportunités et réagissant aux demandes politiques et aux pressions des groupes d'intérêt plutôt qu'en planifiant a priori des stratégies de politique industrielle globales.
Du reste, cela a conduit à une préférence pour les politiques nécessitant des moyens financiers limités. En conséquence, les transferts directs de fonds, au moyen de subventions à des compagnies particulières, n'ont pas été réalisables, déplaçant les priorités vers les interventions structurelles.

La loi $n^{\circ} 281$ de Mai 1970 autorise les régions à mettre en place des agences financières qui sont dans certains cas - sur la base de stipulations précises contenues dans leurs statuts - désignées comme des "conseils en développement et promotion". De tels pouvoirs permettent aux régions d'intervenir sur plusieurs fronts. Ce qui est fait par une variété de moyens lesquels, quoique sous le contrôle de l'autorité régionale, demeurent hors de portée de l'autorité elle-même en termes à la fois d'administration et de recrutement du personnel, et ce dans la mesure où ils sont légalement considérés comme des initiatives de droit privé.

En fait, les agences financières et les conseils en développement ont été créés dans presque toutes les régions pour accomplir des activités de soutien et de promotion et, dans certains cas, une prise directe d'actions et d'intérêts dans les affaires industrielles locales. Les activités des agences financières régionales, spécialement celles opérant dans les régions à statut "spécial", ont traditionnellement fonctionné comme un soutien aux politiques gouvernementales principalement destinées à sauvegarder l'industrie (incluant la participation à la propriété des compagnies locales, comme dans le cas de Friulia de Friuli région Vénétie Julienne) et, seulement dans 


\section{Pôle Sud $N^{\circ} 5$}

un second temps, de procurer des primes financières.

En Émilie-Romagne, le Comité Régional pour le Développement Économique (Ente Regionale per la Valorizzazione Economica del Territorio, ERVET) était créé par la région en décembre 1973 comme société à responsabilité limitée. Le capital était souscrit par la région Émilie-Romagne, qui est l'actionnaire majoritaire, par des institutions bancaires et par la fédération des chambres de commerce (Unioncamere). La tâche d'ERVET est de mener des études ${ }^{6}$ et de superviser des projets spécifiques, soit directement soit par la participation à des compagnies ad hoc. Le rôle d'ERVET a été réaffirmé par la récente loi qui l'a réformé en 1993 (Bellini, Pasquini, 1996).

En théorie, le rapport entre l'agence et le gouvernement régional est celui entre l'exécuteur et le planificateur : un rôle "technico"-tactique face un rôle "politico"-stratégique. La nouvelle loi réaffirme cette division du travail et insiste fortement sur la transparence du processus de prise de décision, en imposant une allocation de ressources et une procédure de contrôle basées un système rigide de financement de projets. Toutefois, ERVET a traditionnellement été un des contributeurs les plus pertinents aux décisions fondamentales de la politique industrielle régionale d'ÉmilieRomagne. Dans le passé (et il n'y a aucune raison de croire que cela ne puisse pas arriver à nouveau) une grande part de l'élaboration de la politique elle-même était entre les mains d'ERVET, à l'exception de la mise sur agenda générale et la décision finale, mais incluant l'étude et la mise en œuvre de la politique, de même que la construction du consensus autour d'elle. Cela était dû à un certain nombre de raisons : la plus grande flexibilité de l'agence, ses ressources humaines techniquement qualifiées, les contraintes légales pesant sur les politiques régionales. Le plus souvent les politiciens en charge des affaires industrielles ont préféré transmettre les dossiers politiquement sensibles à ERVET plutôt qu'à la bureaucratie régionale. En fait, l'histoire se répète maintenant dans le domaine des relations internationales de la région. Le gouvernement régional étant formellement contraint par le "monopole" national sur les affaires étrangères, ERVET joue le rôle le plus actif pour le tissage de relations internationales et a même mis en place, à travers son agence technologique, l'office de représentation à Bruxelles.

\section{Philosophie et contenus de la politique}

La politique industrielle en ÉmilieRomagne peut être analysée par sa subdivision en deux grands volets. Le premier comprend surtout les politiques pour artigianato et pour la formation professionnelle. Dans ces cas la région est formellement en charge d'une telle juridiction et peut compter sur de substantielles ressources financières. Le processus politique et bureaucratique apparaît comme plus fortement influencé par le besoin d'équilibrer la direction des flux financiers que par des options politiques solides. Dans la plupart des cas les distorsions et risques impliqués par cette attitude ont été compensés par une traditionnelle 


\section{Politique industrielle en Émilie-Romagne}

efficacité bureaucratique, une planification soignée et une réelle équité.

Le second groupe de politiques peut être caractérisé comme la "zone grise" de la juridiction régionale en développement. Ici les initiatives politiques ont nettement adopté des caractéristiques innovatrices. L'exemple le plus important est, de loin, celui fourni par le système ERVET des centres de services.

L'Émilie-Romagne peut se vanter d'avoir joué un rôle important concernant ce type d'initiatives. Cela a eu un fort impact sur la forme de la politique industrielle régionale, qui a, par conséquent, été caractérisée comme principalement structurelle (c'est-àdire avec comme objectif d'influer directement sur les variables stratégiques de l'ajustement structurel des compagnies), non distributive (c'est-à-dire non basée exclusivement sur le transfert des ressources financières), territoriale et coopérative (en ce qui concerne les divers acteurs et corps qui forment le système économique local). En fait, de tels centres envisagent et nécessitent une participation active dans la gestion d'un large éventail d'acteurs comme ceux des affaires, les universités, conseils municipaux et provinciaux, chambres de commerce, et entreprises industrielles.

Ces centres agissent comme des agents collectifs, dont la tâche est de donner une meilleure solution à un problème qui ne peut être abordé par des compagnies individuelles. Bien sûr, ce type de solution n'est pas indifférent à la structure de production : dans la mesure où il propose une certaine voie d'ajustement pouvant être acceptée ou refusée par les compagnies individuelles pour de multiples raisons, il promeut le réajustement de la totalité du système et déclenche des processus de sélection au sein de compagnies existantes. Il contribue, par conséquent, à la reformation des réseaux existants et à la définition de nouvelles hiérarchies et positions dominantes.

Dans ce domaine, il est important d'insister sur l'évolution dans la stratégie d'ERVET. À l'origine (jusqu'au début des années 80 ), les acteurs privés et publics locaux ont porté une grande attention à la crise émergente des districts industriels et la région a essentiellement coopéré avec eux pour développer des initiatives spécifiques, agissant à travers sa propre agence de développement, à savoir ERVET. La mise en œuvre de ces initiatives, comme dans le célèbre cas de CITER, le centre pour le secteur du textile et de l'habillement, localisé à Carpi (Modène ${ }^{7}$ ), avait été rendue possible, ou tout du moins facilitée, par précisément la même concentration territoriale des problèmes relatifs à la réorganisation de la production. Ces problèmes aident ou ont aidé à générer la rencontre simultanée du nécessaire consensus critique ainsi que les ressources financières et humaines avec les besoins, dont l'importance collective est donc concrètement visible. C'est ainsi qu'ont été mis en place le centre pour le secteur des céramiques (Centro Ceramico) en 1976 à Bologne, le sus-mentionné centre pour le secteur des textiles et de l'habillement (CITER) en 1980 à Carpi, et le centre pour le secteur des machines à labourer (CEMOTER) en 1982 à Ferrare.

L'agence de développement a ensuite émis ses propres propositions et a ainsi 


\section{Pôle Sud $N^{\circ} 5$}

acquis une position de protagoniste dans l'établissement de nouvelles structures, dont certaines étaient caractérisées par des services multisectoriels. Les centres de "district", dépendant de leur localisation et du bassin principal d'activités, devaient être intégrés, en d'autres termes, à des centres "régionaux". Ceux-ci étaient situés à Bologne ou, de toutes façons, dans des zones qui n'étaient pas associées à des districts particuliers, avec des utilisateurs uniformément répartis sur le territoire régional. Ainsi, le centre du secteur de la chaussure (CERCAL) a été établi en 1983 à San Mauro Pascoli, dans la province de Forli. Le centre pour le matériel agricole (CESMA) en 1983 à Reggio Emilia ; le centre métallurgique (CERMET) et l'agence régionale pour le transfert de technologies (ASTER) en 1985 à Bologne; le centre pour le secteur de la Construction (QUASCO) en 1986 à Bologne et le centre pour la soustraitance dans le secteur ingénierie mécanique (RESFOR) en 1987 à Parme. CERMET, ASTER et, avec un bien moindre succès, RESFOR ont été le type même des agences "régionales-horizontales". Cette tendance fut ensuite confirmée par l'établissement, en 1988, de SVEX, un centre dédié à la promotion à l'export, et celui, en 1989, de FIT, une agence spécialisée dans la garantie financière des innovations technologiques des $\mathrm{PME}^{8}$. Enfin, l'initiative la plus typiquement locale, l'Agence Polo Ceramico, a été établie à Faenza, en 1988.

Dans les années récentes, parallèlement à la complexe structure des centres de services, une nouvelle génération d'instruments, méritant considération, a également émergé. Les deux instances qui caractérisent le mieux ce concept de "nouvelle génération" sont :

- la loi régionale pour la promotion de la qualité industrielle de 1992 ;

- la loi régionale sur les entreprises innovantes de 1994.

Il faut dire que ces lois ne constituent pas une expérience totalement nouvelle en Italie. La Lombardie a joué le rôle pionnier dans ce domaine. Elles ne sont pas ensuite dénuées de contradictions : leur préparation a ainsi mobilisé le lobby des associations d'entreprises sur le plan de la recherche de financements, bien au delà de l'importance réelle des fonds disponibles. Elles peuvent néanmoins incarner une voie de développement possible, particulièrement pertinente dans la perspective d'un accroissement des pouvoirs conférés aux régions. Il est donc intéressant de noter :

- leur caractère plus thématique que sectoriel, et dont l'impact dépend donc au moins autant de l'importance accordée à certaines variables-clef d'ajustement et de croissance que du soutien actuel apporté aux entreprises ;

- le lien établi entre le transfert financier à des entreprises individuelles et d'autres instruments de politique comme, par exemple, le système des centres de service.

Le gouvernement régional a également été impliqué pour la première fois dans la planification du programme européen Objectif 2, pour la période 1994-1999. Bien que limitée du double point de vue géographique et financier, cette participation est hautement instructive et influente en termes 


\section{Politique industrielle en Émilie-Romagne}

de modèles organisationnels et de techniques de programmation.

Dans la même perspective d'un accroissement des pouvoirs, on peut noter une tendance générale qui peut conduire à un rôle plus affirmé de la région (son administration comme ses agences) dans le soutien des PME, et à une approche plus consciente et systématique des problèmes structurels de l'industrie régionale. Cette tendance est marquée par des actions innovantes comme les initiatives pour la mise en réseau inter-régional, qui ont pour objectif explicite d'encourager les interdépendances économiques structurelles avec le Mezzogiorno. Une telle tendance n'est qu'en partie contradictoire avec la faible participation de l'Émilie-Romagne aux quelques missions que la loi $\mathrm{n}^{\circ} 317$ de 1991 sur les PME avait confiées aux régions.

Récemment, plusieurs initiatives locales ont conduit à l'établissement ou à des propositions concrètes pour la création de pôles ou parcs scientifiques et technologiques. Le caractère local de ces initiatives, c'est-à-dire leur indépendance à l'égard de l'ERVET ou d'autres agences régionales, doit être signalé. La région n'a ouvertement joué un rôle de soutien que dans un seul exemple, par l'implication de l'ERVET et l'élaboration d'une loi ad hoc. Cela a été le cas de POSTER (Pôle Scientifique et Technologique d'Émilie-Romagne) à Bologne, une initiative locale qui se présentait elle-même d'envergure et de vocation plus régionales que locales, bien qu'elle n'ait jamais été gérée comme telle en réalité. POSTER fut finalement liquidé. Les propositions pour une loi régionale, renforçant les pouvoirs de la région, furent abandonnées. Quoiqu'il en soit, l'enjeu des relations subsidiaires entre l'ERVET, en tant qu'agence de développement régional, et ces pôles reste d'actualité.

\section{L'évaluation de la politique industrielle en Emilie-Romagne}

Au début des années 1990, à la faveur de la prise de conscience des performances peu brillantes des économies régionales dans les années 1980, un pessimisme sans discernement et simpliste se substitua à la foi inébranlable dans la qualité du "modèle". Une référence rituelle s'installa quant à la "crise" des districts industriels, censée induire une relative perte de validité du système territorial en tant que tel. Aussi symbolique que surprenant que cela puisse paraître, la zone Carpi-Reggio Emilia fut intégrée à la liste des territoires en restructuration industrielle, au sens de l'Objectif 2 des Fonds structurels européens.

La pertinence de la définition usuelle de l'Émilie-Romagne comme typique de la Troisième Italie s'est clairement affaiblie. Pour un nombre croissant d'analystes et de praticiens, ce modèle de développement émilien - comme alternative historique au modèle de développement économique italien dominant - est apparu moins important, autant pour la politique que pour l'analyse, que la complémentarité et le degré d'intégration à la micro-région de la Vallée du Pô (la "Padanie") pour définir les contraintes et opportunités d'une croissance continue. 


\section{Pôle Sud $N^{\circ} 5$}

Personne cependant ne discute la contribution à la croissance économique de la stabilité sociale et politique sur ce territoire et la relative efficience des administrations locales. Néanmoins, le cercle vertueux entre culture civique et développement économique est apparu de plus en plus comme une bonne explication pour le passé mais, quant à l'avenir, de peu de secours pour affronter la sévérité des nouveaux problèmes émergents :

- le retard de modernisation du système infrastructurel, mettant en lumière les limites de la gouvernance locale et régionale ;

- le sérieux enjeu environnemental, montrant que les districts industriels ne sont en aucune manière moins "sales" que les zones de grandes entreprises;

- le problème social lié au chômage (surtout celui des jeunes) et - une radicale nouveauté dans le contexte émilien - au flux important d'immigration, en particulier depuis des pays extra-communautaires.

La culture politique elle-même a changé. Les valeurs, les mythes et les stéréotypes de la Soft Révolution italienne - incluant une idéologie de l'efficience supérieure du marché opposée à l'inefficience irrémédiable des gouvernements - ont été importés, avec un notable degré de généralisation et de manière assez irrespectueuse des composantes de la tradition elle-même.

Il n'est donc pas surprenant que la politique industrielle ait été la cible d'une série de polémiques concernant à la fois la gestion des politiques établies que les arbitrages entre différents instruments (en général : services versus subventions). En fait, les arguments critiques et défensifs ont été caractérisés par l'absence quasitotale d'évaluation sérieuse des politiques existantes et projetées, à l'exception de considérations, certes notables mais marginales, sur l'efficience bureaucratique dans la mise en œuvre des fonds publics et des analogies simplistes entre indicateurs budgétaires dans les centres de services et les firmes privées. Malgré plusieurs discussions préalables, propositions et tentatives, le gouvernement régional lui-même échoua dans tout processus d'évaluation sérieuse et ne saisit pas l'opportunité de nourrir le débat de références fondées en réalité".

Le déficit d'évaluation est d'autant plus sérieux qu'il est objectivement difficile d'attribuer à des politiques maniant des ressources si limitées un impact indiscutable sur l'économie. En conséquence, évaluer la politique industrielle émilienne nécessite l'articulation d'arguments surtout qualitatifs, portant sur le réglage apparent d'initiatives individuelles sur les principales tendances de l'ajustement structurel de l'économie.

Malgré quelques initiatives décevantes, un tel réglage s'est surtout accompli avec les centres de services. Il est dès lors important d'isoler l'évaluation historique de ces expériences, indéniablement positives, d'un jugement sur leur reproductibilité potentielle à l'avenir.

Tout d'abord, ce jugement conduit sans doute à de nouvelles exigences d'efficience des centres eux-mêmes et du système pris globalement. Bien sûr, la différenciation des centres - par les secteurs auxquels ils se réferent, leur période d'établissement, leurs 


\section{Politique industrielle en Émilie-Romagne}

choix organisationnels, les conditions socio-politiques dans lesquelles ils opèrent - rend extrêmement difficile (et potentiellement arbitraire) des comparaisons standardisées (notamment basées sur des ratios d'autofinancement) de leurs performances.

Ensuite, ce jugement devrait être relié au problème du caractère rarement innovant de l'industrie en Émilie-Romagne. Dans les années récentes, cette conviction a donné des arguments de poids aux initiatives de parcs scientifiques et technologiques. Quoiqu'il en soit, la principale expérience (le pôle scientifique et technologique de Bologne) a clairement raté sa mise en relation avec les structures productives régionales, alors que son impact sur les PME était une des attentes majeures, quoique probablement déplacée, des acteurs impliqués (la région, les gouvernements locaux, les associations, l'université).

Le système des centres de services n'a donné qu'une réponse partielle à ce problème du "chaînon manquant" dans le transfert de technologies, bien qu'existent des opportunités significatives de développement sur d'autres thèmes : les systèmesqualité ; la standardisation et la certification ; l'internationalisation. On pourrait globalement identifier une voie de développement des centres de services dans l'évolution du caractère des origines (un secteur, une zone, un thème) vers un rôle "ouvert" de mise en réseau intelligent des structures industrielles locales, où une quantité d'activités d'information et de services complexes, diversifiées (mais reliées), pourrait être fournie aux entreprises. Dans cette perspective, les centres de service peuvent incarner un compromis entre les dimensions globales et locales des politiques technologiques : les premières tendent à l'émergence de structures technocratiques, au moins de niveau régional; les secondes à la territorialisation des activités de services, comme outil de proximité et donc d'efficacité à l'égard des entreprises.

Enfin, comme nous l'avons continuellement affirmé dans cet article, l'évaluation de la politique industrielle en Italie requiert une connaissance précise des contraintes institutionnelles particulières qui se sont imposées à ses options stratégiques. Ce n'est que dans les années récentes que l'on peut situer le début d'une phase de transition vers une appropriation plus complète de la totalité des outils de politique industrielle. Les implications de cette transition demeurent, malgré tout, pour une large part inexplorées. Les régions auront à affronter non seulement le problème de la gestion d'une quantité de ressources significativement plus grande, mais aussi un besoin plus important d'intégration et de coordination à l'intérieur de l'appareil régional et avec les entités extérieures telles que les associations d'entreprises, les chambres de commerce, les universités, etc. Une recherche d'intégration devra également émerger (et cela semble en cours) avec les branches régionales d'activités nationales, comme l'Institut National du Commerce International et le Conseil National de la Recherche.

Dans ce scénario, le problème des instruments est majeur pour plusieurs raisons. D'une part, la capacité de constituer uné 


\section{Pôle Sud $N^{\circ} 5$}

orientation innovante par rapport aux expériences nationales et subnationales antérieures ouvre la voie à de nouvelles approches. En théorie, elle pourrait permettre de dépasser les limites et les contre-indications d'une politique exclusivement basée sur les subventions. D'autre part, ces mêmes limites et contre-indications peuvent tout à fait demeurer valides en pratique, à condition que les subventions induisent une mutation dans l'allocation de ressources, hors des politiques de service. En fait, les pressions dans ce sens existent et sont très puissantes. Il est important de noter que, dans le même temps, la région manque d'outils de financement plus flexibles et discrétionnaires, tels que la prime ou la suppression d'impôt pour soutenir des projets d'investissement.

\section{La politique industrielle décen- tralisée : leçons émiliennes}

Nos remarques conclusives peuvent débuter par l'enjeu de la transposabilité de l'expérience de l'Émilie-Romagne. La question n'est pas nouvelle : l'idée même que l'Émilie-Romagne constitue un modèle implique, quoique dans un sens très simplifié et souvent instrumental, que la "recette" puisse être imitée et adaptée à des contextes différents. Quoiqu'il en soit, les chercheurs locaux ont souvent désappointé les nombreux voyageurs à Bologne et ses environs en mettant en évidence le fait que le modèle dépend de racines profondes, d'un héritage historique que l'on peut difficilement considérer comme reproductibles.

Nous ne croyons pas que l'on doive laisser la question de la transposabilité sans réponse précise, dans une impasse entre des généralisations hyper-simplifiées et des descriptions stériles de spécificités locales. Ce qui peut être reproduit d'Émilie-Romagne n'est pas un modèle général et intégré de croissance mais plutôt :

- une indication de priorité, analytique comme politique ;

- une boîte à outils d'expériences spécifiques d'action publique, et leurs technicités appropriées.

Premièrement, comme nombre d'auteurs étrangers l'ont déjà pertinemment démontré, le principal enseignement venant d'Émilie-Romagne devrait être l'importance clef des préconditions sociales et politiques du développement économique (Cooke, Morgan, 1991; Amin, 1994). Ceci implique que l'action publique donne une priorité effective à l'ingénierie sociopolitique de l'espace public territorial, plutôt que de reposer simplement sur l'efficience technique dans la reproduction des politiques économiques.

Deuxièmement, dans la mesure où les politiques publiques sont concernées, l'Émilie-Romagne dispose d'un nombre d'expériences instructives dont on peut proposer la reproduction dans différents contextes, en particulier dans le domaine des services structurels aux PME. La transférabilité de politiques spécifiques a déjà été tentée avec succès, puisqu'une référence explicite au cas émilien a été faite par des responsables politiques dans nombre de régions européennes (au Danemark et dans la Communauté autonome de Valence, par exemple). 


\section{Politique industrielle en Émilie-Romagne}

Quoiqu'il en soit, nous suggérons que les leçons émiliennes ne doivent pas être limitées à des "transferts d'items". En particulier, on devrait mieux analyser le processus d'adaptation en cours. Nous pensons que le dilemme fondamental de l'ÉmilieRomagne, une fois assurée la disponibilité de la totalité des instruments, est de savoir si cela doit conduire seulement à une homogénéisation de la politique industrielle régionale vis-à-vis des politiques nationales (les premières, d'une échelle plus réduite, étant assimilées aux secondes) ou bien à une dimension toujours spécifiques des politiques régionales. Pour l'Italie, c'est une question importante : dans le premier cas, cela donnerait des arguments aux critiques et doutes à propos des coûts et risques de la fragmentation politique; dans le second cas, cela pourrait renforcer une politique industrielle à plusieurs niveaux, reposant sur le principe de subsidiarité.

En fait, les politiques industrielles décentralisées sont une caractéristique commune aux relations État-industrie dans les économies industrielles avancées, avec l'exception partielle du Royaume-Uni. L'interprétation d'un tel phénomène ne permet pas de synthèse aisée, avant tout parce que son résultat n'est pas un mouvement direct vers une quelconque politique industrielle "fédérale", mais plutôt vers des politiques entrecroisées, un système d'échanges politiques complexes entre différents types d'acteurs. Les débats actuels en Italie autour du "néo-régionalisme" ou du "quasi-fédéralisme" vont dans le même sens (Bellini, 1996).

Le régionalisme en politique industrielle ne peut pas cependant être réduit à une simple réaction à la fameuse surcharge gouvernementale face aux enjeux de l'ajustement industriel. Cette explication est bien sûr pertinente : on peut en effet attendre des acteurs les plus proches des réalités de terrain une plus grande disponibilité et une meilleure sensibilité aux problèmes, et une efficience supérieure dans la recherche de solutions.

Le cas de l'Émilie-Romagne devrait pourtant indiquer que cette analyse ne rend compte que d'une partie de la réalité. En fait, de tels arguments achoppent sur l'auto-perception grandissante des économies locales et régionales. La littérature économique italienne sur les districts industriels, en particulier, a largement contribué à mettre en évidence le caractère "localisé" des processus de production (Beccattini, Rullani, 1993). Dans une perspective de politique publique, ceci implique que la décentralisation ne peut être interprétée comme une simple réduction d'échelle du processus de politique publique et des organisations. La légitimité de la politique industrielle décentralisée ne provient pas de la possibilité d'actions plus efficientes ou mieux ciblées, qui ne feraient que reproduire, à un plus petit niveau, les politiques industrielles des Etats-nations. Elle découle au contraire de la possibilité d'ajouter aux politiques industrielles traditionnelles des dimensions, des outils et des objectifs potentiellement originaux.

Compte tenu également du rôle de l'héritage historique, l'expérience italienne, avec le cas de l'Émilie-Romagne, montre à quel point ces dimensions nouvelles peu- 


\section{Pôle Sud $N^{\circ} 5$}

vent être vastes et importantes. Les expériences telles que les centres de services permettent ainsi de mettre l'accent sur des objectifs spécifiques de politique publique : la mise en réseau des petites entreprises, l'accroissement des atouts permanents d'une région et l'intégration locale et globale des systèmes productifs.

L'économie politique du régionalisme et du localisme comporte aussi des risques. Il n'y a pas seulement, encore un fois et même s'il est important, un risque de désappointement à l'égard de l'inefficience des gouvernements subnationaux ${ }^{10}$. Des échecs de politiques plus fondamentaux et graves sont aussi envisageables : une interconnaissance faiblement enracinée entre les membres du réseau; le déclin des identités territoriales lié au processus d'internationalisation; les conflits que la détresse économique peut engendrer dans une zone et que des politiques innovantes sélectives peuvent, à leur manière, exacerber (Bianchi, Bellini, 1991).

\section{Notes}

1. Le rôle de Bologne a pris de l'ampleur depuis la fin de la deuxième guerre mondiale, mais la ville n'a jamais totalement assumé le profil de "cité-capitale" de la région. En fait, la structure urbaine régionale a le plus souvent été interprétée comme un "système métropolitain" de cités de taille moyenne interdépendantes, suivant le modèle de la Ruhr. Cette interprétation a été officiellement admise dans l'organisation territoriale de la région.

2. Cette liste est adaptée de Bianchi, Gualtieri, 1990.

3. Pour une discussion plus détaillée, $c f$. Bellini, 1989 et 1990 (pour une version anglaise réduite).

4. Cf. Cooke, 1996.

5. Pour une discussion de ces arguments, of. la cinquième partie de cet article.

6. Dans les autres régions elles sont confiées à des instituts de recherche spécialisés.

7. Au début des années 70 le district industriel de Capri était caractérisé par la présence d'un très grand nombre de petites compagnies, hautement exposées aux pressions de la compétition. L'idée de CITER (mis en place en 1980) résulte de l'expérience de la formation professionnelle. Le problème crucial de ce domaine était identifié comme découlant de la faible performance des compagnies locales sur le marché : en tant que suiveurs du marché, ils opéraient dans les segments à faible valeur-ajoutée du marché, avec des produits de qualité médiocre. L'une des ressources nécessaires à un positionnement différent sur le marché résidait dans la disponibilité de flux d'informations constants et fiables en ce qui concerne les tendances de la mode. Cette ressource informationnelle était essentielle à la stratégie d'augmentation du contenu de "mode" des produits : le centre l'a fournie aux compagnies comme un "club good" bon marché, avec l'information technologique correspondante, l'information sur le marché et, plus tard, des occasions pour une innovation originale (une station de travail pour styliste, développée par CITER, avec la base de données complète du travail du centre). Bien sûr, le centre, dont l'activité qualifiée est mondialement reconnue, n'a pas été un agent neutre dans le développement du district de Carpi. En fournissant un tel flux d'information qualifiée, CITER rendit explicite les problèmes communs d'ajustement et déclencha la sélection d'un groupe d'innovateurs et de dirigeants potentiels à l'intérieur de la zone.

8. Les expériences de SVEX, qui empiétaient ouvertement les activités établies de promotion à l'export de la Chambre de Commerce et d'Industrie, de RESFOR et de deux autres initiatives mineures (CETAS, 


\section{Politique industrielle en Émilie-Romagne}

Centre pour la formation des techniciens des pays en voie de développement, et SPOT, pour la promotion de l'industrie métallurgique) ont échoué dans leurs objectifs et ont pris fin. FIT a été progressivement pris en charge par l'ERVET lui-même. Les domaines non stratégiques ont été également abandonnés, comme IDROSER, transformée en Agence pour l'Environnement.

9. Le manque de procédures d'évaluation adéquates, s'il en est, n'est pas du tout une caractéristique propre à l'Émilie-Romagne, mais est partagé par toutes les administrations publiques italiennes, au niveau national comme à l'échelle régionale.

10. Si nous acceptons qu'il y ait toujours une relation directe entre le niveau de développement économique et la qualité du gouvernement, la décentralisation peut déclencher un cercle vertueux pour des zones avancées et un cercle vicieux pour des zones moins développées. Ce risque a été mis en évidence dans le débat sur l'Italie du Sud, de même qu'à travers la résistance du méridionalisme traditionnel à de nouvelles dévolutions de pouvoir aux régions (Trigilia, 1992).

\section{Références}

Amin A., "Globalization and the Local Economy", Atelier international : Industrial Districts and Local Economic Development in Italy : Challenges and Policy Perspectives, Bologne, Mai 1994.

Becattini G., Rullani E., "Sistema locale e mercato globale", Economia e politica industriale, n 80, 1993, pp. 25-48.

Bellini N., "Il PCI ed il governo dell'industria in Emilia Romagna", Il Mulino, $\mathbf{n}^{\circ}$ 5, 1989, pp. 707-732.

Bellini N., "The management of the Economy in Emilia-Romagna : The PCI and the Regional Experience", in Leonardi, Nanetti (eds.), 1990.

Bellini N. (1996), "Regional Economic Policies and the Non-Linearity of History", European Planning Studies, vol. 4, n 1, février 1996, pp. 63-73.

Bellini N., Giordani M. G., Pasquini F., The Industrial Policy of Emilia-Romagna : The Business Service Centres, in Leonardi, Nanetti (eds.), 1990.

Bellini N., Pasquini F., "The Case of ERVET in Emilia-Romagna : Towards a Second-Generation Regional Development Agency", papier présenté au séminaire Regional Development Agencies in Europe : Past Experiences and Future Perspectives, Université d'Aalborg, August 1996.

Bianchi P., Bellini N., "Public Policies for Local Networks of Innovators", Research Policy, $\mathrm{n}^{\circ}$ 20, 1991, pp. 487-497.

Bianchi P., Gualtieri G., "Emilia-Romagna and its Industrial Districts : The Evolution of a Model”, in Leonardi, Nanetti (eds.), 1990.

Brusco S., "The Emilian Model : Productive Decentralization and Social Integration", Cambridge Journal of Economics, $\mathrm{n}^{\circ} \mathrm{G}, 1982$.

Cooke P., "Building a Twenty-first Century Regional Economy in Emilia-Romagna", European Planning Studies, vol. 4, $\mathrm{n}^{\circ} 1$, févier 1996, pp. 53-62.

Cooke P., Morgan K., The Intelligent Region : Industrial and Institutional Innovation in Emilia-Romagna, rapport sur la recherche industrielle régionale $n^{\circ} 14$, Université de Pays de Galles, Collège de Cardiff, 1991.

Franchi M., "Developments in the Districts of Emilia-Romagna", Atelier international : Industrial Districts and Local Economic Development in Italy : Challenges and Policy Perspectives, Bologne, mai 1994.

Leonardi R., Nanetti R. Y. (eds.), The Regions and European Integration. The Case of Emilia-Romagna, Londres, Pinter, 1990.

Pyke F., Becattini G., Sengenberger W. (eds.), Industrial Districts and Inter-Firm Cooperation in Italy, Genève, International Institute for Labour Studies, 1990.

Trigilia C., Sviluppo senza autonomia, Bologne, Il Mulino, 1992. 